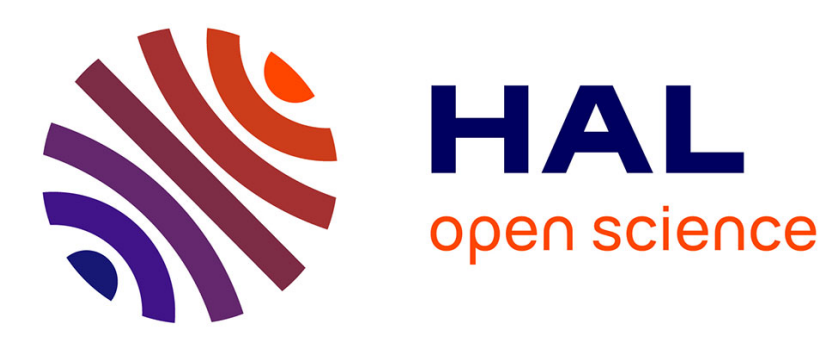

\title{
Dust Detection by Colour Analysis in an Optical of Phonographic Disks Digitisation
}

\author{
Louis Laborelli, Jean-Hugues Chenot
}

\section{To cite this version:}

Louis Laborelli, Jean-Hugues Chenot. Dust Detection by Colour Analysis in an Optical of Phonographic Disks Digitisation. IEEE International Conference on Image Processing, 2007 (ICIP 2007), Sep 2007, San Antonio, United States. pp.25, 10.1109/ICIP.2007.4379945 . hal-01073217

\section{HAL Id: hal-01073217 https://hal.science/hal-01073217}

Submitted on 9 Oct 2014

HAL is a multi-disciplinary open access archive for the deposit and dissemination of scientific research documents, whether they are published or not. The documents may come from teaching and research institutions in France or abroad, or from public or private research centers.
L'archive ouverte pluridisciplinaire HAL, est destinée au dépôt et à la diffusion de documents scientifiques de niveau recherche, publiés ou non, émanant des établissements d'enseignement et de recherche français ou étrangers, des laboratoires publics ou privés. 


\title{
DUST DETECTION BY COLOUR ANALYSIS IN AN OPTICAL METHOD OF PHONOGRAPHIC DISCS DIGITISATION.
}

\author{
Louis Laborelli, Jean-Hugues Chenot \\ INA, Institut National de l'Audiovisuel. 94366 Bry sur Marne, France \\ 1laborelli@ina.fr
}

\begin{abstract}
A contactless optical playing device for 78rpm and 33rpm lateral modulation phonographic discs, using structured colour illumination, is presented.

An area of the disc is illuminated by a beam of rays, with colour depending on the direction of incidence, that are reflected by the groove wall towards a camera. In contrast with standard methods that measure the velocity of the groove at a single location, access to the audio signal is directly obtained here from pictures through colour decoding, and the whole height of the groove wall is exploited for interpolating damaged signal. The pixel colour saturation level is related to the diffuse or specular reflection and allows for the detection of occluding dust, useful for automated interpolation.
\end{abstract}

Index Terms - Microscopy, Colour, Audio, Structured Illumination

\section{INTRODUCTION}

Before the introduction of the magnetic tape recorder in the 1950 's, audio recordings were primarily stored on phonographic discs. A lot of these discs are in archive collections. While the pressed discs (commercial) are more or less stable, the direct cut discs are in danger. The purpose of the described machine and algorithm is to mass digitise easily the decaying media, with optical, contactless methods.

\section{OPTICAL, CONTACTLESS DIGITISATION}

During the last century as well as recently, the playing of phonographic disc playing by optical means has attracted considerable efforts [1].

The advantages expected from a contactless optical digitisation were :

1. No friction of a stylus with the groove, and therefore no record wear. The direct cut discs are particularly fragile, since they are soft enough to be modified by the cutter during engraving. A few playbacks with heavy load can irreversibly destroy the audio signal.

This work was financed by the EU project PrestoSpace FP6-507336
2. Lower non linear distortion: when playing mechanically, the elliptical stylus, while very narrow, cannot enter very large curvature places. A non linear distortion called tracing distortion is inevitable. Optical playback exhibits no such limitation.

3. Lower level of noise, clicks and crackle

4. Avoiding the burden of selecting the right stylus.

The existing research or commercial devices generally obtained good results on the two first points, but progress on the third one is strongly needed. The choice of incoherent illumination in our work instead of a coherent laser beam that creates speckles, is a first step in the reduction of surface noise.

The patent from Stoddard [2], later used in the commercial machine Finial or ELP (Japan) uses the reflection of a laser spot sent to the groove, and reflected toward a PSD (Position sensitive device), an analogue sensor able to determine the precise orientation of a reflected ray. One single point of each groove wall is sampled to get local orientation, but no confidence information is propagated outside for further interpolation of obstructed or deformed wall.

The work from Stotzer and al. [3] is based on a preliminary capture of a disc image to a large scale, high resolution photographic image. An ad-hoc scanner was build to digitise the picture in polar coordinates, and to measure the position of the limits of the groove walls. Numerical differentiation of the tracked curves is used to recover the audio signal.

Fadeyev and Haber [4] have tested the use of several 3D digitisers for the optical playing of disc and cylinders. They have built a prototype of a 2D machine, based on similar principles, ignoring the information present at the surface of the groove wall, and concentrating only on the boundaries.

\section{AGEING OF DIRECT CUT DISCS (ACETATES)}

Most of the historical discs storing radio recordings are of direct recording types, also called acetates, or "lacquers". For instance at INA, the audio archive keeps more than 270000 direct-cut acetates, originating from the 30's to the 50' radio broadcasts. A large proportion of these discs have never been saved on a more recent media, and are often unique. Such 
discs are very fragile, since they are made of two different materials: a plastic-like coating onto which sound was engraved, applied to a stable core made of aluminium.

Even under the best storage conditions, acetate discs are exuding a greasy white powder that creates many physical obstacles to a mechanical or optical play. This powder is most likely the result of the degradation of the plasticisers like dibutyl phthalat (DBP), camphor or castor oil.

With less plasticisers, the acetate coating can shrink and crack like an old painting, and later begins to lift off as it is being played. Such cracked records are very difficult or nearly impossible to play, since the stylus cannot follow a groove, as the retraction can misalign the path on both sides of a crack. The image processing method has the potential to solve this problem.

To remove the dust, the operator plays the disc with different needles to scrap it from the groove, and after a few passes, the disc gets clean and playable. There is still the risk of damaging the groove surface during the cleaning. Other techniques of wet cleaning are advised to dissolve the fatty acid, but already fragmented acetates are too fragile to be handled in a vacuum record cleaning machine.

\section{PRINCIPLE OF OPERATION OF THE PLAYING MACHINE}

We designed an optical machine to play back lateral modulation phonographic records where the groove has a radial deviation speed of which is proportional to the level of the recorded audio signal. The combination of rotational (tangential) speed of the record and of the radial speed of the chiselling mechanism determined a local angle of the groove. The tangent of the groove angle therefore varies in proportion to the recorded sound signal. The measurement of this angle by lighting up one of the faces of the groove that behaves like a mirror, through a structured light, will allow to recover the recorded sound information.

The invention consists in encoding the incident light using colours, so that each point of the image of the groove presents a colour that corresponds to the incident ray beam colour reflected by the groove wall toward the camera, the colour depending only on the angle of the groove wall. This is a form of Rheinberg colour illumination, commonly used in microscopy, modified to obtain quantitative results by the use of a continuous colour filter. The idea of using colour coding for angle is well known in structured light computer vision $[5]$.

The main difference with the existing laser player is the simultaneous measurement of many points of the image, since the tracking of the groove is left to the batch processing phase, allowing for more complex software solutions. In contrast with approaches that compute the position of the groove wall interface with the flat surface of the disc, and differentiate it to recover the radial velocity, the proposed device recovers di- rectly the audio information from pixel colour, and therefore requires a much lower resolution for radial velocity estimation. In our experiments, we used a pixel size of $5 \mu \mathrm{m}$ : high frequency modulation is not visible in the deviation, but is evident in the colour modulation. Recovering the complete orientation profile of the cross section groove is useful for discs damaged by previous mechanical playbacks. Such a process virtually divides the signal on each groove wall into a large number of parallel "subtracks", one for each different depth. Instead of measuring the signal on each groove wall over the small contact area of a stylus, this allows to take into account all the significant parts of the groove wall. At the common speed of 60 images/s for a $640 \times 480$ (progressive) camera, the whole disc side could be digitised in a time close to the real duration of the disc (180 s).

\section{PHYSICAL IMPLEMENTATION}

A 5 watt strobing white LED is used as the source of (cold) light of a standard condenser, composed of 2 aspheric lenses of focal $50 \mathrm{~mm}$ and of diameter $75 \mathrm{~mm}$. This large numerical aperture (N.A.) is the key to obtain a large modulation reading ability. The bundle of coloured rays is produced by a coloured filter printed with a hue varying pattern, placed at the exit pupil. The condenser and filter, visible on the figure 1 , are projecting a $13 \times 72$ degrees beam of coloured rays at an incidence of 45 degrees with the disc plane. After reflection on a small mirror, an area on the disc of $2 \mathrm{~mm}$ by $2 \mathrm{~mm}$ is digitised by a colour Bayer type CCD camera fitted with a 1:1 macro lens. The disc is positioned on a computer controlled translation and rotation table.
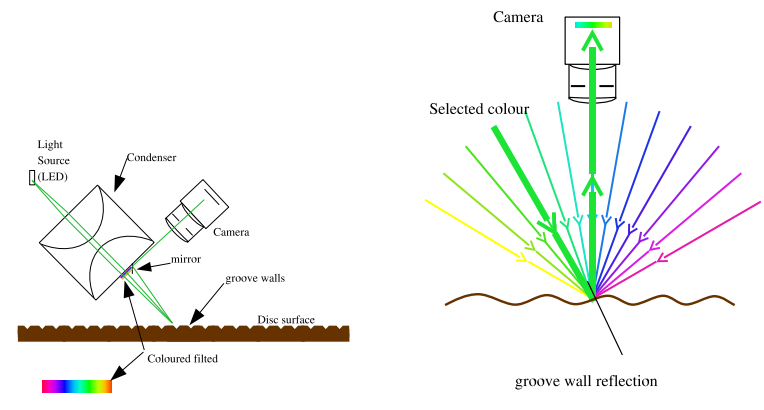

Fig. 1. Device Schematics

\subsection{Modulation range abilities}

A 78rpm disc groove deviation is normally limited by the standard to an angle of $14 \mathrm{deg}$, but a variation of up to $20 \mathrm{deg}$ can be expected. At the viewing incidence of about $45 \mathrm{deg}$, the apparent maximum angle is $\arctan (\tan (20) * \sqrt{2} / 2)=$ $14.4 \mathrm{deg}$. As the angle is doubled by the reflection on the disc wall, the ray entering the camera can come from a maximum half angle of $29 \mathrm{deg}$. (min N.A : 0.49). 


\section{DEBRIS AND DUST DETECTION}

Due to the method of illumination, the rays projected on the disc surface are seen by specular reflection, and the ray colour (hue) and saturation are usually preserved. If some contaminant like the plasticiser degradation product obstructs the view, our hypothesis is that the received light will be less saturated. Indeed, the desaturation is caused by a mixing of different colours coming from different directions and diffused. If the dusty pixels are averaged with the good reflections, they will contaminate the measurements.

\section{GROOVE TRACKING AND SIGNAL EXTRACTION ALGORITHM}

The first step is tracking the groove by computing the centroid of luminance on a window centred around previous location $x$. Pixels collected from a given spatial distance from this centre are kept if they are above some luminance threshold. The second step is the classification of correct signal (specular reflection) versus pixels originating from contaminants (diffuse reflection). This is done by looking at at the image after conversion from RGB to Saturation.

A grey scale dilation of the saturation channel $\mathrm{S}$ by a $3 \times 3$ MIN function kernel is computed, and strongly unsaturated pixels are removed completely. The remaining dilated saturation levels will be used as a weighting map (WM) in the averaging of each RGB pixel over each groove wall profile (i.e. at constant $t$ ). This step is the computation of the weighted mean for each channel $\mathrm{R}, \mathrm{G}, \mathrm{B}$ at each time position $t$, while the sum of this weighting map acts as a temporally varying indicator of the confidence $C(t)$ we have in the data.

\subsection{Interpolation on temporal RGB by adaptive filter}

Only a small number (or even zero) noisy pixels will be participating in the weighted average in a dust occluded or abrasion damaged groove. To correct this effect, the third step is a temporally adaptive box averaging of the RGB data, using a simplified version of the normalized correlation described in [6]. In our implementation the temporal box average of each pixel channel $R(t)$ is computed, weighted by the confidence $C(t)$, and divided by the box average of the confidence $C(t)$. $F(t)$ can be a different kernel than a box.

$$
\tilde{R}(t)=\frac{(C(t) R(t)) * F(t)}{C(t) * F(t)}
$$

Depending of the local confidence, the filtering kernel $F(t)$ width grows up locally to collect enough confidence $C(t)$ (within a given radius limit). This is a locally adaptive averaging filter with variable width $(V W(t))$ which interpolates data where needed.

The interpolated RGBs are then converted to Hue, and represent now the extracted signal from this groove segment which is merged with signals from other images into the global signal at the correct time position using confidence weighting. A global audio signal is extracted at a very high sampling rate (200-300 Ksamples/s), in parallel with a new composite confidence track. This confidence is thresholded to detect the failure of the interpolation first stage due to very wide gaps to activate a standard autoregressive interpolator. The corrected signal is resampled to standard sampling rates and a suitable linear filtering post-accentuation curve is finally applied.

\subsection{RGB to HSI conversion formula}

The following formulas were used for converting the input RGB to HSI (Hue, Saturation, intensity) model :

$$
\begin{aligned}
& H=\arctan \left(\frac{\beta}{\alpha}\right), S=\sqrt{\alpha^{2}+\beta^{2}}, I=(R+G+B) \\
& \text { with }: \alpha=\quad R-\frac{1}{2}(G+B), \beta=\frac{\sqrt{3}}{2}(G-B)
\end{aligned}
$$

The filter printing process is not able to get a constant saturation filter, as seen from the camera. This can degrade the detection method, and generate false alarms. In order to correct this problem, the average saturation was measured for each hue level on a clean sine wave test record and used to compensate the saturation $\mathrm{S}$ before it is used.

\subsection{Colour calibration}

The colour optical filter was printed on a Kodak 8650 dye sublimation printer, in linear mode, as close to the digital file as possible. A hue variation following a tanh curve of the position was computed in order to devote more precision to the centre of the scale, and to saturate gracefully. In RGB space, the colour filter colour distribution is a near complete rainbow-like circle.

As the obtained hue is the result of a process involving the emission spectrum of the LED, the absorption spectrum of the dyes used in the colour rainbow filter, in the Bayer RGB filters of the camera and the spectral sensitivity of the CCD, the instantaneous response is deviating from the tanh curve. A polynomial function is applied to the signal to compensate for the non-linearity in $\mathrm{H}$. This function is obtained by cumulative distribution function (CDF) matching with a vinyl sine wave test record [7].

\section{RESULTS}

Figure 2 (A) is a picture showing some captured grooves from an acetate dusty disc.

The image shows grooves of nearly $150 \mu \mathrm{m}$ wide. Abrasion traces from previous mechanical plays are clearly visible. It is difficult to see on the printed grey-level image the modulation, since the filter is made to make images as constant in luminance as possible. On a colour printing, the colours change depending on the orientation. Debris from plasticiser degradation can be seen. 
In (B) there is a close-up on the upper left part, after conversion to hue level. The modulation is visible, but contaminated by dust particles. In (C) it can be seen that the saturation is a good indicator of the presence of dust, shown in dark shade. (D) is a composite image showing: the rightmost tracked groove (a), the weighting map WM (b), the detected dust (c), the curve $V W(t)$ of the width of adaptive averaging filter larger in dusty areas (d) and the adaptively filtered extracted signal (e).

It can be visually verified that the debris detection method is efficient, with a low proportion of missed pixels. False detection rate is less critical, since the image redundancy will allow to fill the gaps. Objective comparison is difficult, since the original signal is unknown as well as the current state of irrecoverable abrasion of the disc. On audio listening, nearly all the clicks have been removed. Figure 3 is a comparison between the method that does not try to detect the dust before averaging the pixel and measuring the colour, and the method described in this paper, where clicks are removed while preserving the signal. Further details on bandwidth and SNR measurements for clean discs are provided in the paper [8].

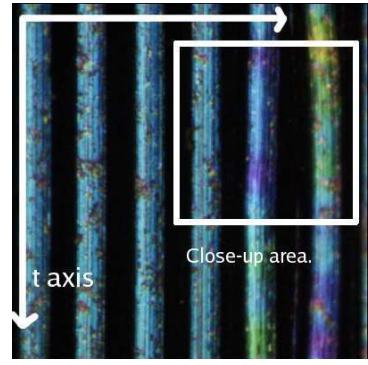

(A) Original image

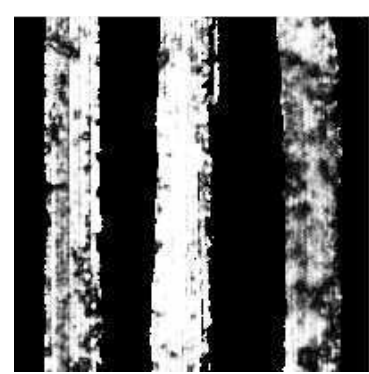

(C) Saturation channel

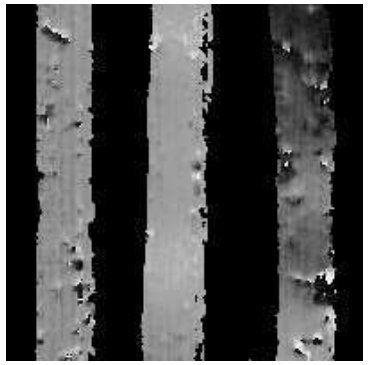

(B) Hue channel

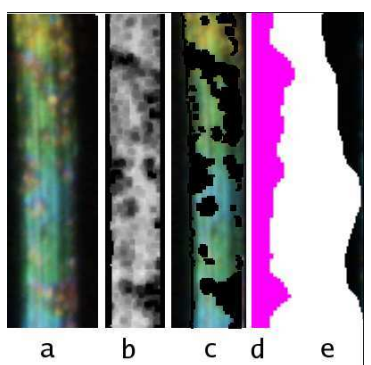

(D) Dust pixels, signal
Fig. 2. Conversion of the rightmost groove to signal

\section{CONCLUSIONS}

An optical method for multiple subtracks digitisation of phonographic discs was described. One of the main drawback of existing methods was the sensitivity to dust contamination. This problem was reduced by a simple, colour saturation based detection of dust, and an adaptive low pass filtering of the image

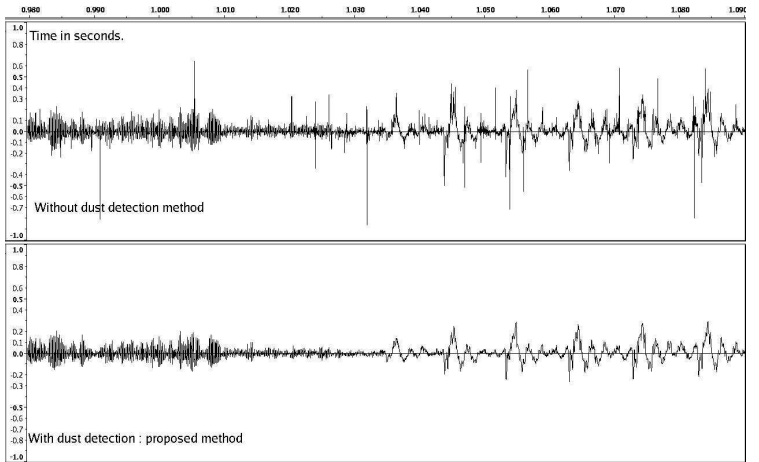

Fig. 3. Comparison of signal decoding: clicks are removed.

data. The tracking and correction of fractured discs grooves remains an open subject.

\section{REFERENCES}

[1] G. Brock-Nannestad, "The attraction of optical replay of mechanical recordings," in 20th AES International Conference (September), 2001.

[2] R. E. Stoddard, "Us4870631 patent: Optical turntable system with reflected spot position detection," 1989.

[3] S. Stotzer., O. Johnsen, F. Bapst, and R. Ingold, "Groove extraction of phonographic records," in DAS06 7th IAPR Workshop on Document Analysis Systems, 2006, pp. 529540.

[4] V. Fadeyev and C. Haber, "Reconstruction of mechanically recorded sound by image processing," Journal of the Audio Engineering Society, vol. 51, pp. 1172, Dec. 2003.

[5] J. Salvi, J. Pages, and J. Batlle, "Pattern codification strategies in structured light systems," Pattern Recognition, vol. 37, no. 4, pp. 827-849, April 2004.

[6] H. Knutsson and C.-F. Westin, "Normalized and differential convolution: Methods for interpolation and filtering of incomplete and uncertain data," in Proc. of IEEE CVPR'93, 1993, pp. 515-523.

[7] R. Balchandran and R. J. Mammone, "Non-Parametric Estimation and Correction of Non-Linear Distortion in Speech Systems," in ICASSP, 1998, vol. 2, pp. 749-752.

[8] L. Laborelli, J-H. Chenot, and A. Perrier, "Non contact phonographic discs digitisation using structured colour illumination.," in 122th AES International Convention (Vienna), 2007. 